\title{
The F-actin filament capping protein CapG is a bona fide nucleolar protein
}

\author{
Thomas Hubert ${ }^{1}$, Katrien Van Impe ${ }^{1}$, Joël Vandekerckhove, Jan Gettemans * \\ Department of Medical Protein Research, VIB, B-9000 Ghent, Belgium \\ Department of Biochemistry, Ghent University, Faculty of Medicine and Health Sciences, Albert Baertsoenkaai 3, B-9000 Ghent, Belgium
}

\section{A R T I C L E I N F O}

\section{Article history:}

Received 8 October 2008

Available online 18 October 2008

\section{Keywords:}

Actin

CapG

Nucleolus

Ran

GTPase

\begin{abstract}
A B S T R A C T
Actin works in concert with myosin I to regulate the transcription of ribosomal genes in the nucleolus. Recently, nucleolar actin has been shown to be active in its polymeric form raising the question how actin dynamics is regulated in the nucleolus. Here, we show that the actin capping protein CapG localizes in the nucleolus of cultured cells. CapG transport to the nucleolus is an active and ATP-dependent process. Association of CapG with the nucleolus requires active RNA Polymerase I transcription. In addition, we show that activated Ran GTPase, an interaction partner of CapG, is also transported to the nucleolus. A constitutively active Ran mutant promotes CapG accumulation in the nucleolus indicating that CapG transport to the nucleolus can be supported by Ran. Our results suggest that filamentous actin in the nucleolus might be regulated by actin binding proteins such as CapG.
\end{abstract}

(c) 2008 Elsevier Inc. All rights reserved.
The nucleolus is the cellular compartment where rDNA genes are transcribed, rRNA is processed and ribosome subunits are assembled [1]. Besides additional recently discovered functions such as cell-cycle progression, proliferation and stress response, ribosome biogenesis remains the primary function of the nucleolus. Indeed, the nucleoli are specifically formed around clusters of rDNA genes at the end of mitosis. Moreover, the structure of the nucleolus is specially organized in concentrically arranged domains for the production, processing and assembly of ribosome components [2].

Nuclear actin has been reported to bind directly to all three RNA polymerases and to regulate their transcriptional activity [3]. Nuclear actin binding proteins such as N-WASP and Arp2/3 are associated, in combination with actin, to RNA Polymerase II and modulate its transcriptional activity [4,5]. Furthermore, it has been suggested that the actin polymerization function of these proteins plays an important role in this process [4,5]. In the nucleolus, actin and myosin I act cooperatively to regulate RNA Polymerase I-mediated transcription [6]. Importantly, actin in its filamentous form and the motor function of myosin I are required for proper functioning of this complex [7].

CapG is an actin filament capping protein of the gelsolin family [8]. Its capping activity is activated by calcium and inhibited by membrane polyphosphoinositides but unlike Gelsolin, CapG does not sever actin filaments [8]. CapG also localizes to the nucleus

\footnotetext{
* Corresponding author. Address: Department of Medical Protein Research, VIB, Ghent University, Faculty of Medicine and Health Sciences, Albert Baertsoenkaai 3, B-9000 Ghent, Belgium. Fax: +32 92649490.

E-mail address: jan.gettemans@ugent.be (J. Gettemans)

1 These authors contributed equally to this work.
}

[9] and we recently showed that CapG is imported in the nucleus by the transport receptor NTF2 and Ran GTPase, a major regulator of nucleo-cytoplasmic transport [10]. The nuclear form of CapG has specifically been shown to promote cell invasion [11]. Interestingly, CapG interacts with both the cytoplasmic (GDP-loaded) and nuclear (GTP-loaded) form of Ran [10]. The nucleotide cycle of Ran is responsible for directionality of transport across the nuclear envelope [12]. Additionally, Ran has been predicted to localize in the nucleolus by a nucleolar proteome study [13].

Proteins involved in ribosome biogenesis, including rDNA transcription, rRNA processing and ribosome assembly, rapidly shuttle between the nucleoplasm and the nucleolus [14]. To evaluate the energy requirements of transport between the nucleoplasm and the nucleolus, an in vitro system has been conceived analogous to the in vitro import assay used for nucleo-cytoplasmic transport analysis $[10,15]$. In contrast to the in vitro import assay where digitonin is used to selectively permeabilize the plasma membrane, leaving the nuclear membrane intact, this assay includes CHAPS in the import reaction mix, which additionally results in permeabilization of the nuclear envelope [16]. Addition of recombinant protein under different energy conditions allows detailed analysis of the energy requirement of transport to the nucleolus. Using a comparable assay, it has been demonstrated that p53 translocates to the nucleolus in an ATP hydrolysis-dependent manner [17].

Here, we demonstrate that endogenous CapG is a nucleolar protein. This localization is dependent on active transcription of ribosomal genes by RNA Polymerase I. In detergent-permeabilized cells, exogenously added CapG translocates to the nucleolus upon addition of ATP but not GTP, revealing that CapG transport to the nucleolus is an active process. Importantly, the localization of CapG 
in the nucleolus, at the center of emerging transcripts, suggests a role for CapG in transcription. Finally, our results suggest that Ran GTPase, a factor directly involved in CapG nuclear import, may assist CapG in nucleolar translocation. Altogether, we propose that actin binding proteins such as CapG might regulate the actin polymerization status in the nucleolus.

\section{Materials and methods}

Plasmids and antibodies. CapG was subcloned in the pEGFP-N1 vector (Clonetech) [9] and in the pcDNA3.1/V5-His vector (Invitrogen). Ran and RanQ69L were subcloned in the pEGFP-C1 vector (Clonetech). EGFP-RanT24N was obtained using the Quikchange mutagenesis kit (Statagene). $\mathrm{His}_{6}$-tagged RanQ69L was a kind gift of D. Görlich (ZMBH Heidelberg, Germany). Ran wild-type was generated from RanQ69L using the Quikchange site-directed mutagenesis kit (Stratagene, La Jolla, CA). Rabbit anti-V5 antibody and mouse monoclonal anti-B23 antibody were obtained from Sigma-Aldrich (St. Louis, USA), mouse monoclonal anti-V5 antibody was obtained from Invitrogen (Merelbeke, Belgium), mouse monoclonal anti-bromouridine antibody, Alexa 488-conjugated goat anti-mouse antibody, Alexa 488-conjugated goat anti-rabbit antibody and Alexa 594-conjugated goat anti-mouse antibody were from Molecular Probes, mouse anti-Ran antibody was from BD Biosciences. Rabbit polyclonal anti-CapG antibody was affinity purified [11]. Rabbit anti-gelsolin antiserum has been described previously [18].

Cell culture and transfection. MCF-7 cells were maintained at $37{ }^{\circ} \mathrm{C}$ in a humidified $10 \% \mathrm{CO}_{2}$ incubator and grown in RPMI
1640 (Gibco BRL Life Technologies) supplemented with 10\% fetal bovine serum, $100 \mu \mathrm{g} / \mathrm{ml}$ streptomycin and $100 \mathrm{IU} / \mathrm{ml}$ penicillin. HeLa, MDCK and HEK293T cells were grown in DMEM with $10 \%$ fetal bovine serum, $100 \mu \mathrm{g} / \mathrm{ml}$ streptomycin and $100 \mathrm{IU} / \mathrm{ml}$ penicillin. HeLa cells were transiently transfected using lipofectamine reagent (Invitrogen) according to the manufacturer's instructions. HEK293T cells, seeded on rat tail collagen-coated coverslips, were transfected using calcium phosphate. For actinomycin D (actD; Sigma-Aldrich) and $\alpha$-amanitin (Sigma-Aldrich) treatment, HEK293T cells were plated on collagen-coated coverslips, allowed to recover for $24 \mathrm{~h}$ and stimulated with $0.05 \mu \mathrm{g} / \mathrm{ml}$ actinomycin D or $25 \mu \mathrm{g} /$ $\mathrm{ml} \alpha$-amanitin in DMEM for $3 \mathrm{~h}$ before processing for immunofluorescence microscopy. For bromouridine incorporation, cells were incubated with DMEM containing $20 \mathrm{mM}$ bromouridine (SigmaAldrich) for $1 \mathrm{~h}$ at $37^{\circ} \mathrm{C}$.

Recombinant proteins. Purification of Myc-CapG-V5-His 6 has been previously described [10]. Human $\mathrm{His}_{6}-\mathrm{RanQ69L}_{\mathrm{L}}$ and $\mathrm{His}_{6}{ }^{-}$ Ran wild-type were expressed and purified as described [19]. Gelsolin was purified as described [20]. RanQ69L protein was GTPloaded by incubation with $1 \mathrm{mM}$ GTP in the presence of $15 \mathrm{mM}$ EDTA for $60 \mathrm{~min}$ at room temperature. Magnesium chloride was added to a final concentration of $30 \mathrm{mM}$. The loading reaction was performed immediately prior to the import reaction.

In vitro import assay. Cell permeabilization and in vitro import reactions were carried out as described [15]. Briefly, MDCK-AZ cells were grown on glass coverslips for $48 \mathrm{~h}$. Cells were washed in transport buffer $(20 \mathrm{mM}$ Hepes, $\mathrm{pH} 7.3,110 \mathrm{mM}$ potassium acetate, $5 \mathrm{mM}$ sodium acetate, $2 \mathrm{mM}$ magnesium acetate, $2 \mathrm{mM}$ DTT, $0.5 \mathrm{mM}$ EGTA and protease inhibitors) and then treated
A

HEK

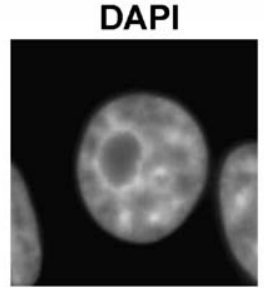

MCF-7
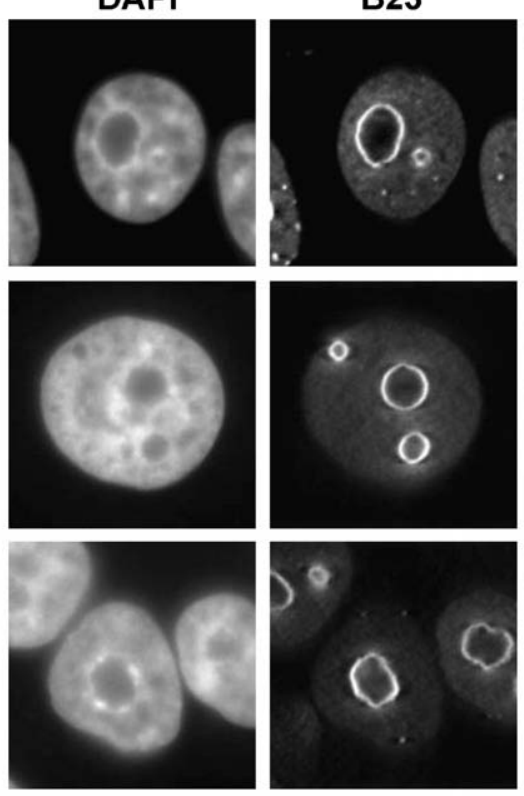

B
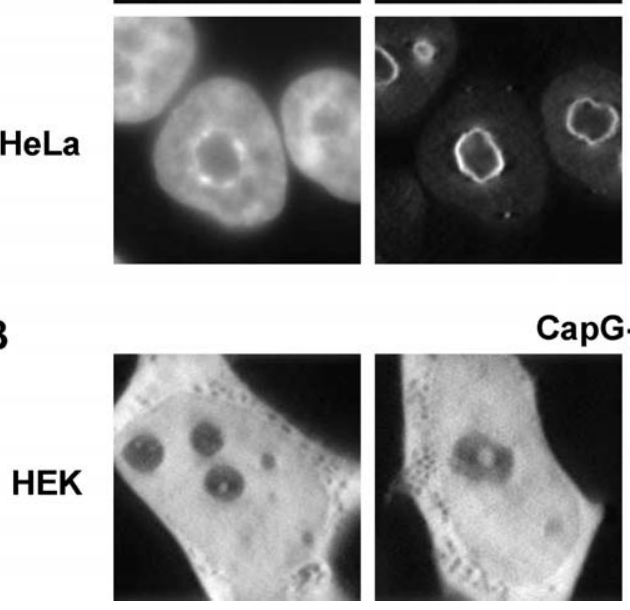
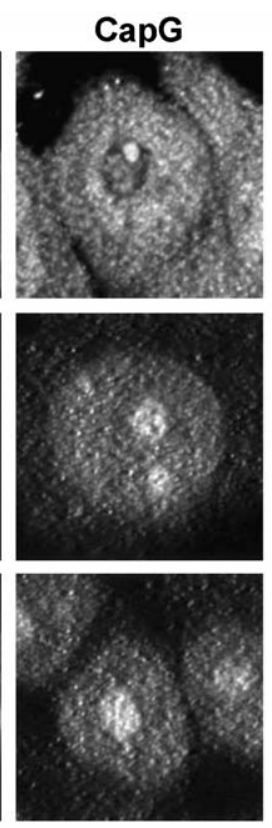

\section{CapG-EGFP}
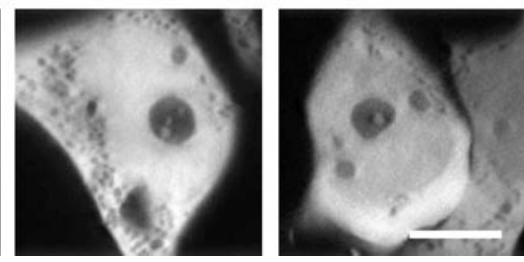

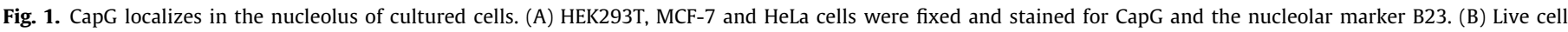
imaging of HEK293T cells expressing CapG-EGFP. Scale bar $=10 \mu \mathrm{m}$. 
with digitonin $(20 \mu \mathrm{g} / \mathrm{ml})$ for $4 \mathrm{~min}$ on ice. After permeabilization, the cells were washed twice with transport buffer and inverted on top of $50 \mu \mathrm{l}$ import reaction mix containing $4 \mu \mathrm{M}$ transport substrate and an energy-regenerating mixture (energy) (1 mM ATP, $0.1 \mathrm{mM}$ GTP, $5 \mathrm{mM}$ creatine phosphate and $20 \mathrm{U} / \mathrm{ml}$ creatine phosphokinase) and incubated at $30^{\circ} \mathrm{C}$ for $30 \mathrm{~min}$. Permeabilization of the nuclear envelope was accomplished by adding one-tenth volume of $20 \mathrm{mM}$ Tris, $\mathrm{pH} 7.0$, containing $10 \%$ glycerol and $1 \%$ CHAPS to the import reaction. In the condition where extra ATP or GTP was present, $8 \mathrm{mM}$ ATP or GTP was additionally added to the import mixture. Cells were washed twice in transport buffer and further processed for immunofluorescence microscopy.

Immunostaining and immunofluorescence microscopy. Cells were washed with PBS, fixed with 3\% paraformaldehyde for 20 min at room temperature and permeabilized with $0.2 \%$ Triton $\mathrm{X}$ 100 in PBS for $5 \mathrm{~min}$. Paraformaldehyde was neutralized with $0.75 \%$ glycine for $20 \mathrm{~min}$. Cells were then blocked in $1 \%$ BSA in PBS for $30 \mathrm{~min}$ and incubated with primary antibody for $1 \mathrm{~h}$ at $37^{\circ} \mathrm{C}$ or overnight at $4{ }^{\circ} \mathrm{C}$. Cells were washed in PBS, then incubated with secondary antibody (Alexa 488-conjugated goat anti-rabbit or Alexa 594-conjugated goat anti-mouse; Molecular Probes) and 4,6-diamidino-2-phenylindole (DAPI; Sig$\mathrm{ma}$ ) for $30 \mathrm{~min}$ at room temperature. Following immunostaining, samples were analyzed using a Carl Zeiss Axiovert $200 \mathrm{M}$ Apotome epifluorescence microscope $(63 \times 1.4 \mathrm{NA}$ oil objective $)$ equipped with an Axiocam cooled CCD camera and processed using Axiovision software (Zeiss).

\section{Results}

CapG localizes to the nucleolus

Immunofluorescence microscopy of paraformaldehyde-fixed cultured cells revealed that in addition to the previously reported cytoplasmic and nuclear localization, endogenous CapG also localizes in the nucleolus (Fig. 1A). Costaining with B23, a marker for the nucleolar boundary, showed CapG staining inside the nucleolus. To examine the specificity of the CapG staining, we analyzed several cell lines, including HEK293T, HeLa and MCF-7 cells (Fig. 1A). In addition, imaging of EGFP-tagged CapG revealed CapG localization in the nucleoli of live HEK293T cells, excluding a possible fixation artifact (Fig. 1B). Interestingly, CapG-EGFP assembled in compact intranucleolar dots, suggesting that CapG is targeted to a specific compartment of the nucleolus (Fig. 1B).

CapG localization in the nucleolus is dependent on RNA Polymerase I activity

To further develop these findings, we used bromouridine incorporation and immunofluorescence to visualize nascent transcripts in the nucleoplasm and nucleolus. CapG localization in the nucleolus coincided with a region that is depleted in bromouridine-labeled transcripts (Fig. 2A, upper panel, arrowhead), which is characteristic for the nucleolar fibrillar center [21]. Low concentrations of the transcriptional inhibitor of RNA Polymerase I actinomycin D caused CapG to relocalize to perinucleolar sites (Fig. 2A,
A

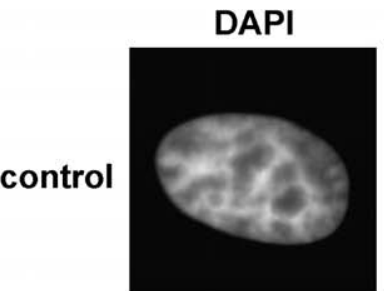

$\operatorname{ctD}$
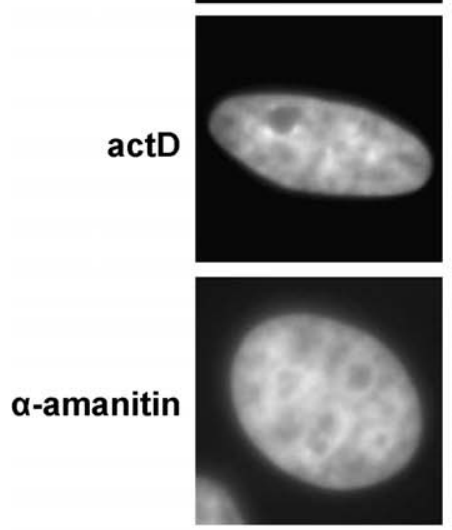

B

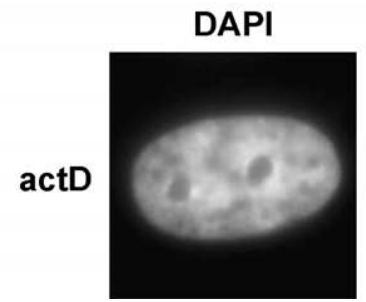

$\mathrm{BrU}$
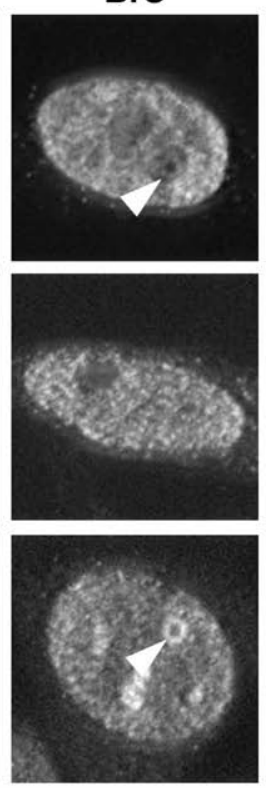

B23

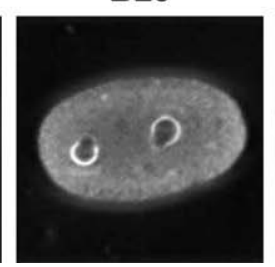

CapG
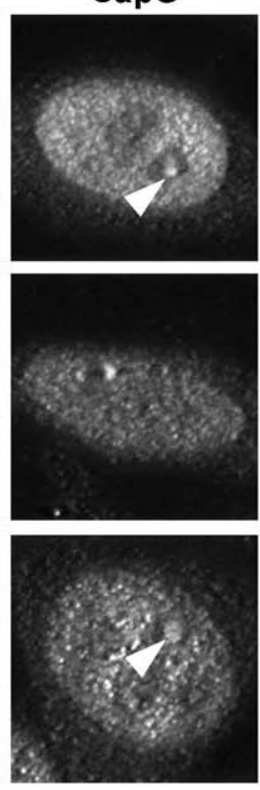

CapG

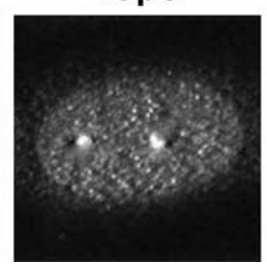

merge
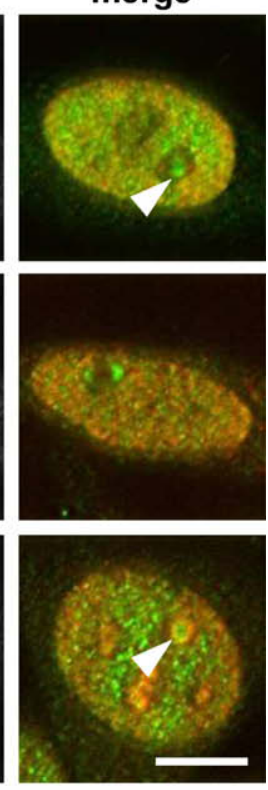

merge

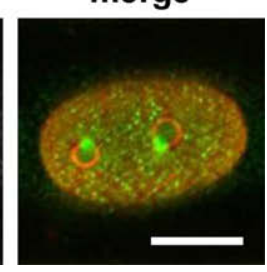

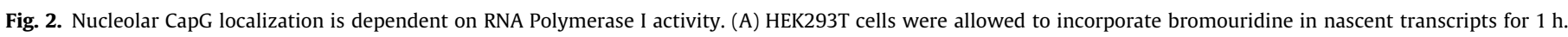

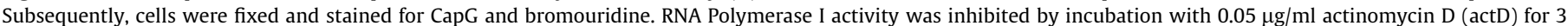

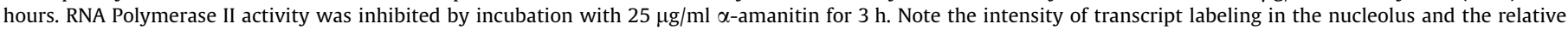
position of the CapG staining. (B) HEK293 T cells were incubated with $0.05 \mu \mathrm{g} / \mathrm{ml}$ actinomycin D for $3 \mathrm{~h}$, fixed and stained for CapG and B23. Scale bar $=10 \mu \mathrm{m}$. 
middle panel and Fig. 2B). In contrast, inhibition of RNA Polymerase II using $\alpha$-amanitin did not influence CapG localization in the nucleolus (Fig. 2A, lower panel). As a result of reduced RNA Polymerase II activity, nucleolar transcripts visualization was improved and CapG was occasionally detected at the center of a ring-shaped pool of nucleolar transcripts (Fig. 2A, lower panel, arrowhead). Thus, the specific localization of CapG in the nucleolar interior is consistent with the rDNA transcription sites.

\section{CapG is actively transported to the nucleolus}

We next used an in vitro assay to analyze the energy requirements for CapG transport to the nucleolus [17,22]. The plasma membrane of MDCK cells was permeabilized with digitonin. Subsequently, recombinant CapG was added to the permeabilized cells under several energy conditions in an import reaction mix containing CHAPS. Under low energy conditions, CapG did not accumulate in the nucleolus (Fig. 3A, upper panel), indicating that CapG transport to the nucleolus is an active process and not the result of passive diffusion. When ATP was added, CapG strongly accumulated in the nucleolus (Fig. 3A, lower panel). By contrast, GTP did not stimulate CapG localization to the nucleolus (Fig. 3A, middle panel). As a control, Gelsolin did not accumulate in the nucleolus upon addition of either GTP or ATP (Fig. 3B).

In a previous study, we reported that CapG interacts directly with Ran GTPase [10]. Since Ran is predicted to localize in the nucleolus by a proteome study (http://www.lamondlab.com/NOP$\mathrm{db} /$ ) and has been reported to accumulate in the nucleolus in in vitro experiments [23], we also tested Ran in our experiments (Fig. 3C). Under low energy conditions, GTP-loaded RanQ69L but
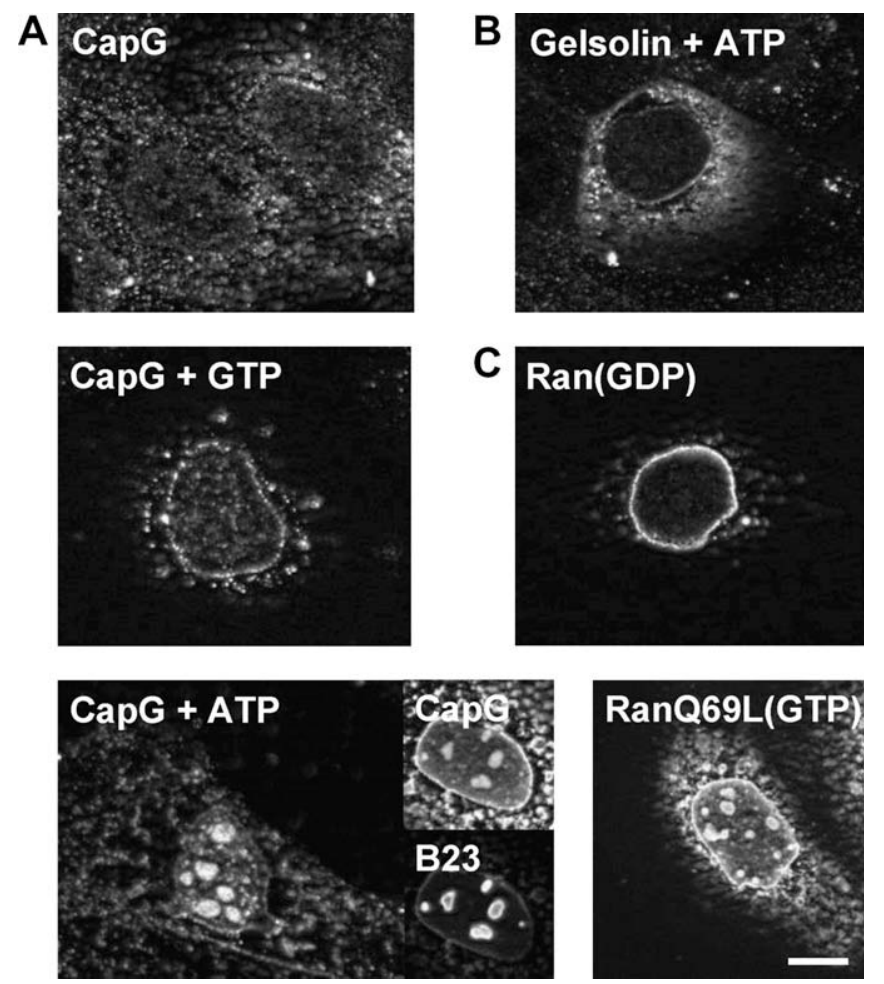

Fig. 3. CapG is actively transported to the nucleolus. GTP- but not GDP-loaded Ran accumulates in the nucleolus. (A) MDCK cells were permeabilized with digitonin and CHAPS and recombinant CapG was added under different energy conditions: $0.1 \mathrm{mM} \mathrm{GTP}+1 \mathrm{mM}$ ATP (upper panel), 8 mM GTP (middle panel), 8 mM ATP (lower panel). The localization of the nucleoli was visualized by a B23 staining. (B) As a negative control, recombinant Gelsolin was added to permeabilized MDCK cells with $8 \mathrm{mM}$ ATP. (C) GDP-loaded Ran or GTP-loaded RanQ69L were added to permeabilized MDCK cells with $0.1 \mathrm{mM} \mathrm{GTP}+1 \mathrm{mM}$ ATP. Scale bar $=10 \mu \mathrm{m}$. not GDP-loaded Ran strongly accumulated in the nucleolus (Fig. 3C), suggesting that the nucleoplasmic but not the cytoplasmic Ran form is able to enter and accumulate in the nucleolus.

\section{RanGTP induces CapG accumulation in the nucleolus}

Since CapG and GTP-loaded Ran accumulate in nucleoli (Fig. 3) and the two proteins interact directly [10], we explored the localization of overexpressed CapG and Ran in function of the nucleotide status of Ran. Therefore, CapG was expressed in HeLa cells in combination with either wild-type Ran, the GTPase-deficient Ran Q69L mutant or the GTP-binding deficient Ran T24N mutant. Wild-type Ran and CapG were uniformly distributed between nucleoplasm and nucleolus (Fig. 4, upper panel). Interestingly, the GTP-loaded Ran Q69L mutant and CapG synergistically accumulated in the nucleolus (Fig. 4, middle panel), suggesting that GTP-loaded Ran is able to target CapG to the nucleolus. In contrast, both the GDP-loaded Ran T24N mutant and CapG were absent from the nucleolus in cells expressing both proteins (Fig. 4, lower panel).

\section{Discussion}

Although filamentous actin in the nucleus has been reported in several occasions, its functional significance has only recently been demonstrated [7,24,25]. Indeed, polymeric actin and the motor activity of nuclear myosin I have been shown to be necessary for the transcription of rDNA genes in the nucleolus [7]. The length of nucleolar actin filaments is probably tightly regulated as are the cytoplasmic filaments. Consequently, one might expect that actin binding proteins regulate the polymerization status of actin in the nucleolus. In this study, we demonstrate that CapG is a likely candidate to fulfill this role. Intriguingly, although actin and myosin I localization on rDNA do not require active RNA Pol I transcription [7], the presence of CapG in the nucleolus does correlate with nucleolar transcriptional activity. This could imply that polymeric actin on rDNA genes needs to be regulated by actin binding proteins in order to be active in transcription.

Since CapG directly interacts with Ran [10] and Ran has been predicted to localize to the nucleolus [13], we tested Ran as well in the permeabilized cell assay (Fig. 3C). As expected, RanGDP, the cytoplasmic form of Ran, was not imported into the nucleolus. In contrast, RanGTP, the nucleoplasmic form of Ran, did accumulate into the nucleolus. Selective entry of GTP-bound Ran into the nucleolus could be confirmed in intact cells by expressing mutants of Ran. Remarkably, CapG and Ran behaved in a similar manner when expressed simultaneously in HeLa cells. A Ran mutant that cannot bind GTP (Ran T24N) was excluded from the nucleolus and in these cells CapG was not localized to the nucleolus either. A Ran mutant that cannot hydrolyze GTP (Ran Q69L) and that is almost exclusively GTP-loaded did accumulate in the nucleolus, as did CapG. These results indicate that CapG can enter the nucleolus together with the GTP-loaded form of Ran.

Our results also confirm that CapG can interact with both RanGDP and RanGTP [10]. This is understandable assuming that Ran is necessary for both CapG entry in the nucleus and CapG import into the nucleolus. Indeed, active transport of CapG to the nucleus requires cytoplasmic GDP-loaded Ran [10,23] whereas active transport to the nucleolus is enhanced by nucleoplasmic GTP-loaded Ran. However, how the interaction between CapG and Ran is regulated irrespective of the nucleotide status of Ran remains unclear. Moreover, the results from the permeabilized cell assay suggest an alternative ATP-dependent translocation pathway to the nucleolus. Clearly, residual Ran in permeabilized cells and addition of GTP are not sufficient for CapG import. Thus, in addition to RanGTP, additional factors may be involved in CapG transport to the nucleolus. 


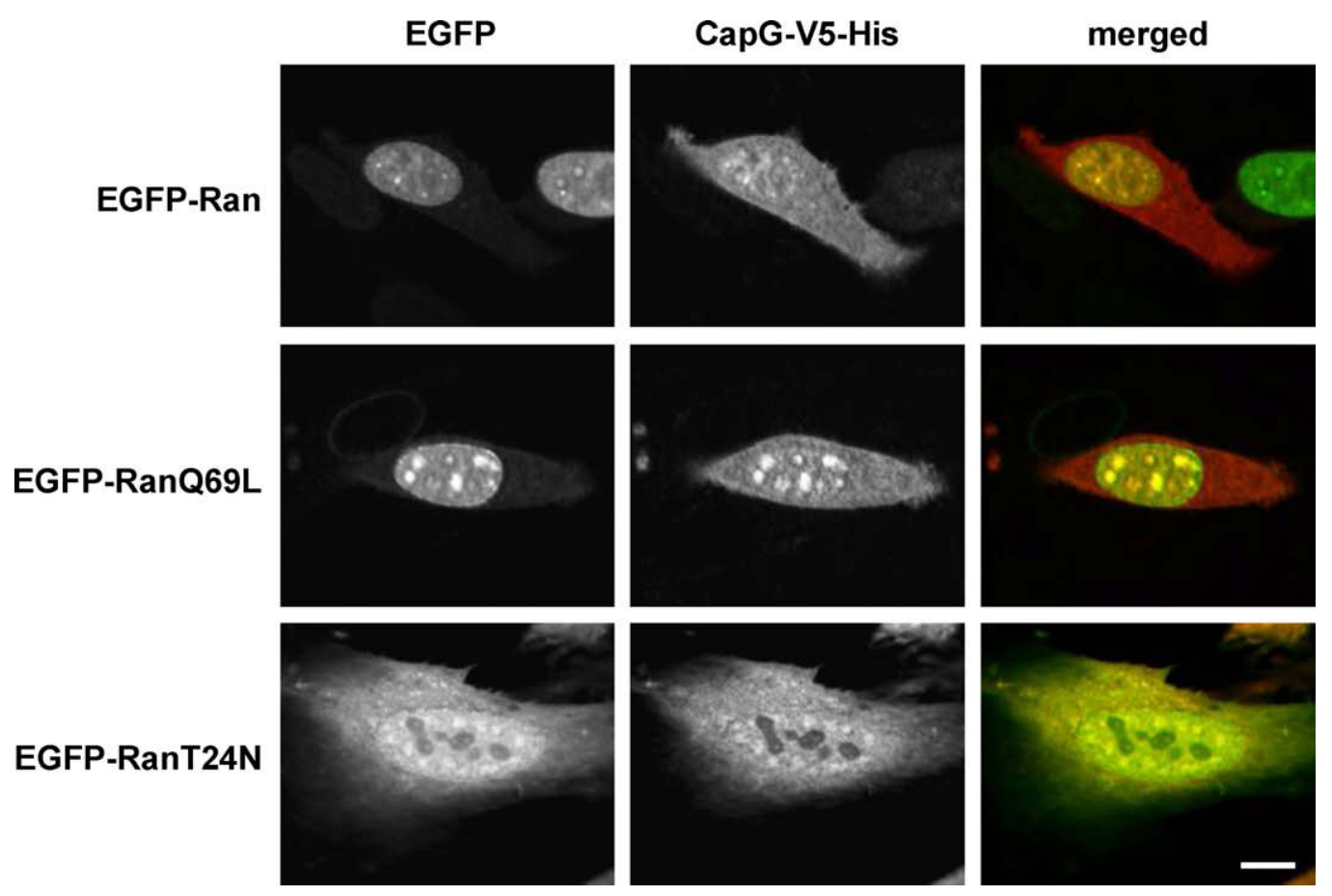

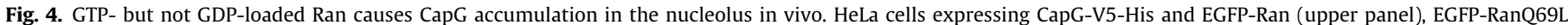
(middle panel) or EGFP-RanT24N (lower panel). Scale bar $=10 \mu \mathrm{m}$.

It is puzzling that RanGTP enters the nucleolus because virtually no endogenous Ran was observed in the nucleolus (data not shown). However, Exportin1/CRM1, an export receptor for nuclear cargos, is predicted to localize in the nucleolus by a proteome study [13]. CRM1 has also been shown to bind Rev (cargo) in the nucleolus [26] and to target other cargos to the nucleolus [27]. In view of these data, and since CRM1 acts in concert with RanGTP to bind cargo, it may not be surprising to detect Ran in the nucleolus. Possibly epitope masking, permeabilization conditions or paraformaldehyde-based fixation of cells prevents Ran staining of the nucleolus. Furthermore, our finding that a Ran mutant which cannot hydrolyze GTP (Ran Q69L) strongly accumulates in the nucleolus suggests that the Ran GTP/GDP cycle is required for Ran to exit from the nucleolus. GTP hydrolysis by Ran is required for cargo to be released from nuclear import complexes [12]. Therefore, in analogy with its role in nucleo-cytoplasmic exchange, it is likely that Ran operates in different modes to support transport of macromolecules between the nucleoplasm and the nucleolus.

\section{Acknowledgments}

RanQ69L cDNA was a kind gift from Prof. Dr. D. Görlich (Universität Heidelberg, Germany). This work was supported by the Concerted Actions Program of Ghent University (GOA), the Interuniversity Attraction Poles (IUAP), the human Frontier Science Program (HFSP) and the Fund for Scientific Research-Flanders (FWO-Vlaanderen).

\section{References}

[1] F.M. Boisvert, S. van Koningsbruggen, J. Navascues, A.I. Lamond, The multifunctional nucleolus, Nat. Rev. Mol. Cell Biol. 8 (2007) 574-585.

[2] I. Raska, P.J. Shaw, D. Cmarko, Structure and function of the nucleolus in the spotlight, Curr. Opin. Cell Biol. 18 (2006) 325-334.

[3] F. Miralles, N. Visa, Actin in transcription and transcription regulation, Curr. Opin. Cell Biol. 18 (2006) 261-266.

[4] X. Wu, Y. Yoo, N.N. Okuhama, P.W. Tucker, G. Liu, J.L. Guan, Regulation of RNApolymerase-II-dependent transcription by N-WASP and its nuclear-binding partners, Nat. Cell Biol. 8 (2006) 756-763.
[5] Y. Yoo, X. Wu, J.L. Guan, A novel role of the actin-nucleating Arp2/3 complex in the regulation of RNA polymerase II-dependent transcription, J. Biol. Chem. 282 (2007) 7616-7623.

[6] N. Fomproix, P. Percipalle, An actin-myosin complex on actively transcribing genes, Exp. Cell Res. 294 (2004) 140-148.

[7] J. Ye, J. Zhao, U. Hoffmann-Rohrer, I. Grummt, Nuclear myosin I acts in concert with polymeric actin to drive RNA polymerase I transcription, Genes Dev. 22 (2008) 322-330.

[8] F.X. Yu, P.A. Johnston, T.C. Sudhof, H.L. Yin, gCap39, a calcium ion- and polyphosphoinositide-regulated actin capping protein, Science 250 (1990) 14131415.

[9] K. Van Impe, V. De Corte, L. Eichinger, E. Bruyneel, M. Mareel, J. Vandekerckhove, J. Gettemans, The nucleo-cytoplasmic actin-binding protein CapG lacks a nuclear export sequence present in structurally related proteins, J. Biol. Chem. 278 (2003) 17945-17952.

[10] K. Van Impe, T. Hubert, V. De Corte, B. Vanloo, C. Boucherie, J. Vandekerckhove, J. Gettemans, A new role for nuclear transport factor 2 and Ran: nuclear import of CapG, Traffic 9 (2008) 695-707.

[11] V. De Corte, K. Van Impe, E. Bruyneel, C. Boucherie, M. Mareel, J. Vandekerckhove, J. Gettemans, Increased importin-beta-dependent nuclear import of the actin modulating protein CapG promotes cell invasion, J. Cell Sci. 117 (2004) 5283-5292.

[12] M. Stewart, Molecular mechanism of the nuclear protein import cycle, Nat. Rev. Mol. Cell Biol. 8 (2007) 195-208.

[13] A.K. Leung, L. Trinkle-Mulcahy, Y.W. Lam, J.S. Andersen, M. Mann, A.I. Lamond, NOPdb: nucleolar proteome database, Nucleic Acids Res. 34 (2006) D218-D220.

[14] D. Chen, S. Huang, Nucleolar components involved in ribosome biogenesis cycle between the nucleolus and nucleoplasm in interphase cells, J. Cell Biol. 153 (2001) 169-176.

[15] S.A. Adam, R.S. Marr, L. Gerace, Nuclear protein import in permeabilized mammalian cells requires soluble cytoplasmic factors, J. Cell Biol. 111 (1990) 807-816.

[16] A. Efthymiadis, H. Shao, S. Hubner, D.A. Jans, Kinetic characterization of the human retinoblastoma protein bipartite nuclear localization sequence (NLS) in vivo and in vitro. A comparison with the SV40 large T-antigen NLS, J. Biol. Chem. 272 (1997) 22134-22139.

[17] O. Karni-Schmidt, A. Friedler, A. Zupnick, K. McKinney, M. Mattia, R. Beckerman, P. Bouvet, M. Sheetz, A. Fersht, C. Prives, Energy-dependent nucleolar localization of p53 in vitro requires two discrete regions within the p53 carboxyl terminus, Oncogene 26 (2007) 3878-3891.

[18] A. Van den Abbeele, V. De Corte, K. Van Impe, E. Bruyneel, C. Boucherie, M. Bracke, J. Vandekerckhove, J. Gettemans, Downregulation of gelsolin family proteins counteracts cancer cell invasion in vitro, Cancer Lett. 255 (2007) 57-70.

[19] U. Kutay, E. Izaurralde, F.R. Bischoff, I.W. Mattaj, D. Gorlich, Dominant-negative mutants of importin-beta block multiple pathways of import and export through the nuclear pore complex, EMBO J. 16 (1997) 1153-1163.

[20] K. Meerschaert, V. De Corte, Y. De Ville, J. Vandekerckhove, J. Gettemans, Gelsolin and functionally similar actin-binding proteins are regulated by lysophosphatidic acid, EMBO J. 17 (1998) 5923-5932. 
[21] P. Hozak, P.R. Cook, C. Schofer, W. Mosgoller, F. Wachtler, Site of transcription of ribosomal RNA and intranucleolar structure in HeLa cells, J. Cell Sci. 107 (Pt. 2) (1994) 639-648

[22] M.H. Wu, C.Y. Lam, B.Y. Yung, Translocation of nucleophosmin from nucleoli to nucleoplasm requires ATP, Biochem. J. 305 (Pt. 3) (1995) 987-992.

[23] K. Ribbeck, G. Lipowsky, H.M. Kent, M. Stewart, D. Gorlich, NTF2 mediates nuclear import of Ran, EMBO J. 17 (1998) 6587-6598.

[24] D. McDonald, G. Carrero, C. Andrin, G. de Vries, M.J. Hendzel, Nucleoplasmic beta-actin exists in a dynamic equilibrium between low-mobility polymeric species and rapidly diffusing populations, J. Cell Biol. 172 (2006) 541-552.
25] S. Zhang, C. Kohler, P. Hemmerich, F. Grosse, Nuclear DNA helicase II (RNA helicase A) binds to an F-actin containing shell that surrounds the nucleolus, Exp. Cell Res. 293 (2004) 248-258.

[26] D. Daelemans, S.V. Costes, S. Lockett, G.N. Pavlakis, Kinetic and molecular analysis of nuclear export factor CRM1 association with its cargo in vivo, Mol. Cell. Biol. 25 (2005) 728-739.

[27] S. Boulon, C. Verheggen, B.E. Jady, C. Girard, C. Pescia, C. Paul, J.K. Ospina, T. Kiss, A.G. Matera, R. Bordonne, E. Bertrand, PHAX and CRM1 are required sequentially to transport U3 snoRNA to nucleoli, Mol. Cell 16 (2004) 777-787. 